\title{
Search for an equilibrium between heat demand and supply in heating systems under a unified heat supply organization
}

\author{
Andrey V. Penkovsky,** \\ ${ }^{1}$ Melentiev Energy Systems Institute of Siberian Branch of the Russian Academy of Sciences (ESI SB \\ RAS), Pipeline Energy Systems Department, 130, Lermontov str., Irkutsk, Russia, 664033
}

\begin{abstract}
Organizational model of the heat energy market on a unified heat supply organization is considered. An equilibrium mathematical model is proposed for the liberalized tariff setting conditions based on a microeconomic monopolistic market model. This mathematical model allows for taking into account energy production and transportation costs as part of a single economic criterion and to determine the supply and demand equilibrium for heat energy. The search for an optimal solution to the developed mathematical model of the unified heat supply organization is based on the univariate relaxation method with the subsequent use of methods of redundant design and simple iterative schemes. The developed mathematical model fully reflect the current "rules of the game" between heat energy producers and consumers and allow a maximum consideration for the interests of all participants of the heat supply process under physical and engineering constraints on heat energy sources and heat networks.
\end{abstract}

\section{Introduction}

There are more than 50 thousand heat markets in the Russian Federation [1] and all of them are controlled by natural monopolies. Most of them represent local heating systems that have branched heat networks and a limited number of heat sources.

Currently, Russia's heat power industry goes toward liberalization and market reforms [2], which suggests abolishment of state control over heat tariffs and establishment of a unified heat supply organization (UHSO) according to the Federal law No.190 «On heat supply» [3]. This unified heat supply organization will make it possible to reduce the heat production costs and to more effectively supply heat to consumers in its service area.

This model of heat supply is being gradually adopted in real practice in urban and rural areas of the Russian Federation. The proposed model suggests the integration of all heating system functions, including heat generation, transportation and sale, to be performed within the unified heat supply organization.

With this heat supply model, the unified heat supply organization should become the owner of all the municipal assets including heat sources and distribution heat networks.

\footnotetext{
* Corresponding author: penkoffsky@isem.irk.ru
} 
Such a merging of the main assets and heat supply control processes shapes the unified heat supply organization as a single seller in the heat market, i.e. a monopolist. Thus, the unified heat supply organization will have a total control of heat supply in the market and market heat price.

The relationships between the market participants in the form of the model of the unified heat supply organization develop according to a certain pattern and imply the following. Based on the forecasts of demand and optimal options of heating system expansion, the unified heat supply organization delivers heat (under medium-term and long-term contracts) to consumers at a price calculated as a sum of a price of heat source heat generation and a price of heat transportation from the heat source to the consumer. In this case, the unified heat supply organization produces the amount of heat that on the one hand would maximize its profit, given physical-technical constraints on heat sources and heat networks, and meet the demand for heat specified by the consumer, and on the other hand would correspond to the consumer wish to pay for this demand.

It is worthwhile to emphasize a classical microeconomic model of monopoly [4-7] among the most widely applied approaches to modeling of the medium-term (or long-term) forecasting of possible

\section{A mathematical model of the Unified heat supply organization}

Heat market in the format of an organizational model of the unified heat supply organization with a great number of sources and heat networks is modeled by a hydraulic circuit. The hydraulic circuit represents a calculated scheme of a real heating system that consists of $m$ nodes and $n$ branches [8]. The hydraulic circuit structure is described by a total coupling matrix $\bar{A}$. The number of rows in this matrix coincides with the number of nodes, and the number of columns - with the number of branches. Hydraulic circuit represents an aggregate of ordered sets, including a set of nodes $-J=\{j: j=1, \ldots, m\}$ that consists of subsets: $J_{h s}$ - heat source, $J_{c o n}$ - consumers and $J_{0}$ - ordinary branching nodes in the scheme; and a set of branches $-I=\{i: i=1, \ldots, n\}$, that show the specified pairwise relations between the nodes.

Modeling of such a system is performed with a certain time interval, that starts with the initial time $\tau_{0}$ (for example, corresponding to a calculated heating load) and finishes with the final (design) time $\mathrm{T}$.

According to [9], the optimal flow distribution in the heat network for each calculated time $\tau$ can have the following form:

$$
\begin{gathered}
\mathrm{Ax}_{\tau}=\mathrm{G}_{\tau}, \\
\overline{\mathrm{A}}^{\mathrm{T}} \overline{\mathrm{P}_{\tau}}=\mathrm{h}_{\tau}-\mathrm{H}_{\tau}, \\
\mathrm{h}_{\tau}=\mathrm{SX}_{\tau} \mathrm{x}_{\tau},
\end{gathered}
$$

where $\mathrm{A}-$ an $(m-1) \times n$ coupling matrix of linearly independent $m$ nodes and $n$ branches; $\mathrm{x}_{\tau}-\mathrm{a}$ vector of flow rates in the network segments $\mathrm{t} / \mathrm{h} ; \mathrm{G}_{\tau}-\mathrm{a}$ vector of mass flow rates at nodes, $\mathrm{t} / \mathrm{h} ; \overline{\mathrm{A}}^{\mathrm{T}}-\mathrm{a}$ transposed coupling matrix of $m$ nodes and $n$ branches; $\overline{\mathrm{P}_{\tau}}-\mathrm{a}$ vector of nodal pressures, mwc; $\mathrm{h}_{\tau}-\mathrm{a}$ vector of losses, mwc; $\mathrm{H}_{\tau}-\mathrm{a}$ vector of real heads, mwc; $\mathrm{S}$ and $\mathrm{X}_{\tau}(n \times n)-$ diagonal matrices of order $n$, including the values of coefficients of hydraulic resistance of branches $s\left(\mathrm{mh}^{2} / \mathrm{t}^{2}\right)$ and absolute values of flow rates in them $\left|x_{\tau}\right|(\mathrm{t} / \mathrm{h})$. 
The equation relating the quantity of heat with mass flow rate of heat carrier is determined by the known relationship [10]:

$$
Q_{j \tau}=c \cdot G_{j \tau} \cdot\left(t_{1}-t_{2}\right)
$$

where $c$ - heating capacity of heat carrier, $\mathrm{GJ} / \mathrm{kg} \cdot{ }^{\circ} \mathrm{C} ; t_{1}$ and $t_{2}$ - temperature of the network water in supply and return pipelines, respectively, ${ }^{\circ} \mathrm{C}$;

Part of nodes in the hydraulic circuit represent the sites where heat sources that have heat output limitations are located, GJ/h:

$$
Q_{j_{-} \min }^{h s} \leq Q_{j \tau}^{h s} \leq Q_{j_{-} \max }^{h s}, j \in J_{h s}, \tau=\tau_{0}, \ldots, \mathrm{T} .
$$

A set of consumers in the heat market can be represented by two subsets: residential consumers and industrial consumers.

Demand of residential consumers for heat $Q_{j \tau}^{r c}$ is determined by the curve of heating load duration. Configuration of this curve is rather well defined by Rossander equation. According to this equation, heating load at each time $\tau$ is determined by the expression [11], GJ/h:

$$
Q_{j \tau}^{r c}=\left[1-(1-r) \cdot\left(\tau / \tau_{h p}\right)(g-r) /(1-g)\right] \cdot Q_{j}^{h l}+Q_{j}^{h w l}, j \in J_{r c}
$$

where $Q_{j}^{h l}$ - calculated heating load, GJ/h; $Q_{j}^{h w l}$ - calculated hot water load, GJ/h; $r$ and $g$ - coefficients of heating load curve unevenness; $\gamma$ - share of hot water load; $\tau_{h p}-$ duration of heating period.

Demand for heat of the industrial consumer $Q_{j \tau}^{I C}$ is modeled by demand characteristic. This characteristic is based on real calculations for individually considered industrial consumers by approximating the retrospective data, given the forecast assessments of heat consumption volumes and heat prices. A more general form of the characteristic can be as follows $[12,13]$, GJ/h:

$$
Q_{j \tau}^{i c}=\varphi_{j}\left(w_{j \tau}\right), j \in J_{i c},
$$

where $\varphi_{j}\left(w_{j \tau}\right)$-demand for heat of the consumer.

Each industrial consumer has their limitations on heat consumption, $\mathrm{GJ} / \mathrm{h}$ :

$$
Q_{j \tau}^{i c} \leq Q_{j_{-} \max }^{i c}, j \in J_{i c} .
$$

Behavior of the unified heat supply organization in the heat market is determined by the amount of profit to be gained as a result of meeting the demand for heat specified by the consumer. In this case, the objective function takes account of both the heat production costs, and the costs related to its transportation to each consumer [14], EUR:

$$
P^{U H S O}=\sum_{j \in J} w_{\tau} \cdot Q_{j \tau}^{h s}-Z^{\text {total }}
$$

where $Z^{\text {Total }}=\sum_{j \in J} Z_{j s}^{h s}\left(Q_{j}^{h s}\right)+\sum_{i \in I} Z_{i \tau}^{h n}-$ total costs related to heat production $\sum_{j \in J} Z_{j s}^{h s}\left(Q_{j}\right)$ and heat transportation $\sum_{i \in I} Z_{i \tau}^{h n}$, EUR; $w_{\tau}-$ heat price or the inverse demand function, EUR/GJ.

The heat production costs of heat sources at any time $\tau$ can be represented by a functional dependence on the amount of heat produced by them. It includes both variable (fuel) and constant (maintenance) costs $[15,16]$ :

$$
Z_{j \tau}^{h s}\left(Q_{j \tau}^{h s}\right)=\alpha_{j} \cdot\left(Q_{j \tau}^{h s}\right)^{2}+\beta_{j} \cdot Q_{j \tau}^{h s}+\gamma_{j}, \alpha_{j}>0, j \in J_{h s}, \tau=\tau_{0}, \ldots, \mathrm{T},
$$


where $\alpha_{j}, \beta_{j}, \gamma_{j}$ - approximation coefficients of heat source cost characteristic.

Heat network costs are determined according to the following analytical dependence [17] and include operating costs and costs of heat carrier pumping in the heat networks:

$$
\sum_{i \in I} Z_{i \tau}^{h n}\left(x_{i \tau}\right)=F_{1}+F_{2} \cdot \sum_{i \in I} x_{i \tau}^{2}\left|x_{i \tau}\right| \cdot s_{i}
$$

where $x_{i \tau}$ - heat carrier flow rate in the $i$-th section of the heating network at time $\tau, \mathrm{t} / \mathrm{h}$; $F_{1}$ - semi-fixed costs, EUR; $F_{2}$ - coefficient at semi-fixed heat network costs, EUR.

Based on the above mentioned, an equilibrium between demand and supply in the heat market for liberalized model of the unified heat supply organization is determined by solving the problem of profit maximization of the organization [14], given constraints.

Find:

$$
\sum_{\tau=\tau_{0}}^{\mathrm{T}} P_{\tau}^{U H S O}=\sum_{\tau=\tau_{0}}^{\mathrm{T}} \sum_{j \in J} w_{\tau} \cdot Q_{j \tau}^{h s}-\sum_{\tau=\tau_{0}}^{\mathrm{T}} \sum_{j \in J} Z_{j \tau}^{h s}\left(Q_{j \tau}^{h s}\right)-\sum_{\tau=\tau_{0}}^{\mathrm{T}} \sum_{i \in I} Z_{i \tau}^{h n}\left(x_{i \tau}\right) \rightarrow \max ,
$$

subject to (1)-(8).

The search for an optimal solution to the developed mathematical model of the unified heat supply organization is based on the univariate relaxation method [18] with the subsequent use of methods of redundant design [19] and simple iterative schemes. The method suggests the reduction of the multidimensional optimization problem to onedimensional one and the use of a stepwise procedure for the improvement of solutions concerning the volumes of heat production by heat sources.

The computational process of search for an equilibrium between demand and supply based on the considered model can be presented graphically. To this end, we will consider a heating system with two heat sources and time interval $\tau=1$ (Fig.1).



$I$ - numbers of heat network sections; $I I$ - numbers of heat network nodes

Fig.1. A calculated scheme of heat supply to consumers.

Graphically, the objective function of the unified heat supply organization is a convex paraboloid with its vertex directed upward (Fig.2 a)). Figure 2 b) illustrates isolines of an objective function that show the area of change in the profit under various combinations of heat production volumes of heat sources. The computational process starts with a zero coordinate toward an increase in the profit of the unified heat supply organization (point A, Figure $2 \mathrm{~b}$ ). Optimal solution for the considered example is obtained already in the $5^{\text {th }}$ 
iteration and corresponds to point $Q^{*}$. The broken line A-B-C-D- $Q^{*}$ represents the computational process trajectory itself.



a)

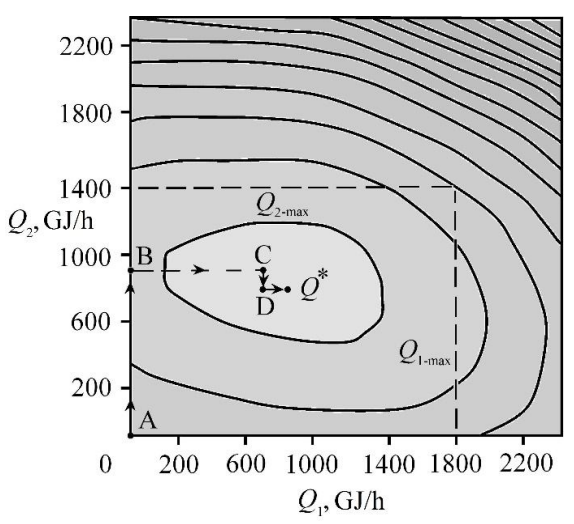

б)

Fig.2. Graphical interpretation of a computational process of search for an optimal solution.

The equilibrium achieved at point $Q^{*}$, represents an optimal solution in terms of the heat volumes produced by the heat sources, equilibrium price of heat production and profit of the unified heat supply organization that corresponds to these values, provided the physical and technical constraints in the heating system are met, and demand equals supply at time $\tau=1$ for the calculated ambient temperature.

\section{Conclusions}

An equilibrium mathematical model is developed for the organizational model of the heat market in the format of the unified heat supply organization. The model is based on the microeconomic model of the monopoly market. This mathematical model makes it possible to take into account the heat production and transportation costs as a single economic criterion. An algorithm is proposed to search for an optimal solution to the mathematical model of the unified heat supply organization. The algorithm is based on the method of univariate relaxation. The heating system with two heat sources was used to exemplify the computation technique of search for a solution.

\section{Acknowledgements}

The research was performed at Melentiev Energy Systems Institute SB RAS in the framework of a scientific projects III.17.4.3, № AAAA-A17-117030310437-4 of the foundation researches program of SB RAS.

\section{References}

1. L.D. Gitelman, V.E. Ratnikov, Energy business (Delo, Moscow, 2006) [in Russian]

2. V.V. Kudryavyi, Energ. Prom-st. Rossii, 2 (2015) [in Russian]

3. Federal Law of the Russian Federation «On heat supply»,190 (2010) [in Russian] 
4. V.P. Busygin, E.V. Zhelobodko, A.A Tsyplakov, Microeconomics - the third level (Novosibirsk State University, Novosibirsk, 2003) [in Russian]

5. J. Tirole, The Theory of Industrial Organization (MIT Press., Cambridge, MA, 1988)

6. D. Carlton, J. Perloff. Modern Industrial Organization. (Reading, MA, AddisonWesley, 2000)

7. P .Belleflamme, M. Peitz, Industrial Organization: Markets and Strategies (Cambridge University Press, Cambridge, UK, 2010)

8. A.P. Merenkov, V.Y. Khasilev, Theory of hydraulic circuits (Nauka, Moscow, 1985) [in Russian]

9. A.P. Merenkov, Mathematical modeling and optimization of heat, water, oil and gas supply system (Nauka, Novosibirsk, 1992) [in Russian]

10. E.Y. Sokolov. District heating and heat networks (Energoizdat, Moscow, 1982) [in Russian]

11. A. Rossander, Die Anwendung der symbolischen Belastungskurven für die Elektrizitätswerke (ETZ, 1913) [in German]

12. P. Vasant, N. Voropai, Sustaining power resources through energy optimization and engineering (Engineering Science Reference, Hershey PA, USA, 2016)

13. V.A. Stennikov, O.V. Khamisov, A.V. Penkovsky, International Journal of Energy Optimization and Engineering, 2 (2013)

14. H. Gravelle, R. Rees, Microeconomics, 3rd Edition, (Prentice Hall, Essex, England, 2004)

15. V.A. Stennikov, O.V. Khamisov, A.V. Penkovsky, Thermal Engineering, 58 (12), 1043, (2011)

16. A.V. Penkovskii, V.A. Stennikov, O.V. Khamisov, Thermal Engineering, 62 (8), 598, (2015)

17. A. V. Penkovskii,V.A. Stennikov, O.V. Khamisov, E.E. Mednikova, I.V. Postnikov, Energy procedia, 105 (2017)

18. E. Shoup, A practical guide to computer methods for engineers (Prentice-hall, England, 1979)

19. E.V. Sennova, V.G. Sidler, Mathematical modeling and optimization of developing heating systems (Nauka, Novosibirsk, 1987) [in Russian] 